\title{
The growth of InAlP using trimethyl amine alane by chemical beam epitaxy
}

\author{
G.O. Munns, W.L. Chen, M.E. Sherwin and G.I. Haddad \\ Center for High Frequency Microelectronics, The University of Michigan, 1135 EECS Building, Ann Arbor, Michigan 48109-2122, USA
}

\begin{abstract}
The growth of InAIP and related compounds such as InGaP lattice matched to GaAs has attracted a great deal of interest for optoelectronic devices emitting in the range from 638 to $700 \mathrm{~nm}$ and for electronic devices such as the heterojunction bipolar transistor. Although some gas source MBE work has been performed in this material system, very little CBE work has been done, largely attributable to the lack of a suitable aluminum source. This is the first report of trimethyl amine alane (TMAA) being used to grow InAIP. TMAA offers advantages of less carbon incorporation and less oxygen sensitivity compared to triethyl aluminum, tri-isobutyl aluminum, or trimethyl aluminum. Trimethyl amine alane has been used to grow AlGaAs HBTs and more recently to grow InAlAs / InGaAs HEMTs by CBE. One of the principal strengths of CBE is its ability to handle phosphorus based compounds efficiently, offering excellent interface control. InAlP films with carbon concentrations below $7 \times 10^{17} \mathrm{~cm}^{-3}$ lattice matched $|\delta a / a|<2 \times 10^{-3}$ with good surface morphology have been grown. Double crystal X-ray diffraction exhibits a single epi-peak with a full width at half max of 46 arc sec with multiple Pendellösung fringes. The epitaxial films are semi-insulating, completely depleted for thicknesses up to $1.6 \mu \mathrm{m}$. Oxygen levels measured by secondary ion mass spectroscopy are comparable to levels measured in InAlAs films $\left(\sim 2.5 \times 10^{18} \mathrm{~cm}^{-3}\right)$ lattice matched to InP. The likely source of this oxygen is the hydride precursor as has been shown for the growth of InAlAs. As the substrate temperature is raised, the films become increasingly indium-rich. Breaking the growth rate down into its constituent binaries indicates an enhanced TMI incorporation rate. The quality of the films as measured by X-ray full width at half max and the surface morphology is extremely sensitive to substrate temperature. A very narrow window exists for the growth of good quality material in the range from 535 to $545^{\circ} \mathrm{C}$.
\end{abstract}

\section{Introduction}

Wide band gap III-V alloys have recently come under inspection for optoelectronic applications. The InGaP/InAlP material system has recently demonstrated excellent laser characteristics [1] and has also shown promise for heterojunction bipolar transistors. The InAIP is of interest because it exhibits the smallest refractive index and largest band gap (although indirect) of any arsenic or phosphorus based alloy which is lattice matched to GaAs. While MOCVD [2], MBE [3], and GSMBE [4] have been used to grow this material system, very few reports of chemical beam epitaxially grown InAIP have appeared [5] despite the ease of phosphorus handling associated with $\mathrm{CBE}$. CBE may also provide interface abruptness advantages over MOCVD and semi infinite sources not found in GSMBE or MBE.

The primary limitation of CBE grown InAlP until now has been the lack of a suitable aluminum precursor. A report of InAlP grown using trimethyl aluminum and triethyl aluminum reported unintentional carbon incorporation in excess of $1 \times 10^{19} \mathrm{~cm}^{-3}$ and highly resistive films [5]. The recent emergence of trimethyl amine alane has previously facilitated the growth of $\mathrm{AlGaAs}$ and InAlAs. The lack of $\mathrm{Al}-\mathrm{C}$ bonds and its relative insensitivity to volatile alkoxide contamination compared to TMAl, TEAl, or triisobutyl aluminum supports the growth of $\mathrm{Al}$ GaAs [6] and InAlAs [7] films comparable in quality to either MBE or MOCVD grown material. 
A series of epitaxial layers have been grown directly on GaAs substrates to investigate the properties of CBE grown InAIP using TMAA.

\section{Material growth}

The epitaxial films were grown for this study in a first generation Intevac/Varian CBE system. Phosphine and arsine ( $99.9995 \%$ no dilution) were used as the group $\mathrm{V}$ presursors, while adduct purified (Air Products) trimethyl indium, triethyl gallium, and trimethyl amine alane served as group III sources. The group $\mathrm{V}$ hydrides were co-injected and thermally cracked at $894-1000^{\circ} \mathrm{C}$ in a low pressure cracking furnace. Previous studies indicated that $894^{\circ} \mathrm{C}$ was the optimum temperature for smooth surface morphology and good electrical characteristics of lattice matched epitaxial InP. No drying filters for the hydrides have been used in these studies.

The TEG and TMAA were coinjected through a low temperature $\left(75^{\circ} \mathrm{C}\right)$ cell using palladium purified hydrogen as a carrier gas. No problems were associated with this combined introduction; although no $\mathrm{Al} / \mathrm{Ga}$ alloys have been grown in this system. After initial oxide desorption, a substrate temperature between 500 and $600^{\circ} \mathrm{C}$ was chosen. Films were typically between 0.5 and 1.6 $\mu \mathrm{m}$ in thickness.

\section{Characterization}

All films were characterized by phase contrast Nomarski inspection and double crystal X-ray diffraction (DCXRD) of lattice constant. Fig. 1 shows a DCXRD scan of a $0.6 \mu \mathrm{m}$ thick film grown on GaAs. It exhibits a single epi-peak with several Pendellösung fringes, a lattice match $|\delta a / a|<2 \times 10^{-3}$ (with no strain correction factor), and a FWHM of 46 arc sec. This compares very favorably to material grown by GSMBE [4]. Using the X-ray diffraction spectra as a basis for comparison; however, a very narrow window in substrate temperature exists for the growth of good quality material in the range $535-545^{\circ} \mathrm{C}$. On either side of this window the X-ray signal at-

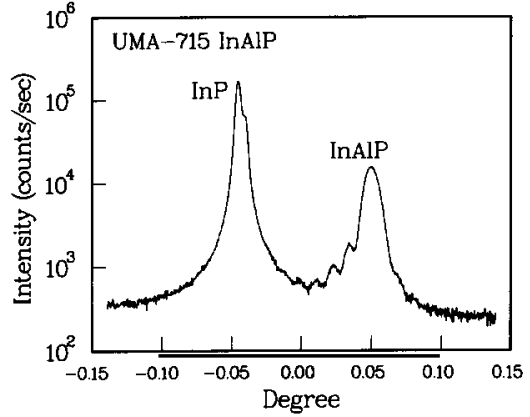

Fig. 1. Double crystal X-ray diffraction of $0.6 \mu \mathrm{m}$ thick InAlP grown on $\mathrm{GaAs}$ at $540^{\circ} \mathrm{C}$.

tributed to the epi-layer widens and the intensity is reduced. This reduction in the $\mathrm{X}$-ray quality tracks with surface morphology which rapidly deteriorates outside the growth window, quickly becoming very rough.

Secondary ion mass spectroscopy indicates the films have carbon concentrations below $7 \times 10^{17}$ $\mathrm{cm}^{-3}$. This is more than one order of magnitude less than previously reported levels in material grown by CBE [5]. Fig. 2 also shows oxygen levels of $\sim 2.5 \times 10^{18} \mathrm{~cm}^{-3}$ and Si concentrations near $1 \times 10^{16} \mathrm{~cm}^{-3}$. The source of this oxygen is the hydrides as previously determined in a parametric investigation of InAlAs [7]; however, it is nearly an order of magnitude higher than that found in InAlAs, probably indicating a higher $\mathrm{O}_{2} / \mathrm{H}_{2} \mathrm{O}$ component in the phosphine. Hall measurements made on this material indicate that it is completely depleted for epi-thickness of more than $1.6 \mu \mathrm{m}$.

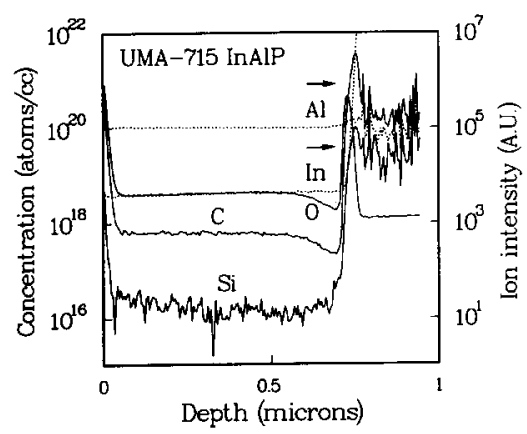

Fig. 2. Secondary ion mass spectra of same sample as shown in fig. 1. 


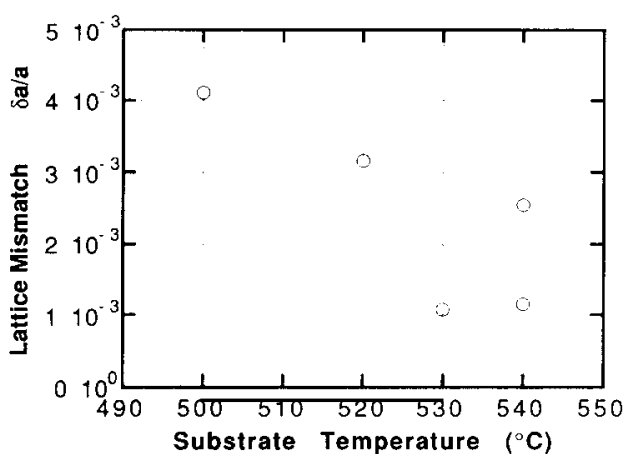

Fig. 3. Lattice mismatch versus substrate temperature for constant hydride cracking temperature, group $\mathrm{V}$ flow and group III flow.

With increasing substrate temperature the films become more indium-rich. Fig. 3 shows a plot of lattice match versus substrate temperature. While there is some scatter in the data, it may be due to variation among substrate holder temperatures which has been estimated to be $\sim 10^{\circ} \mathrm{C}$. Using a selective $\mathrm{HCl}: \mathrm{H}_{2} \mathrm{O}$ facilitated epi-thickness measurement from which growth rates were determined. Using this information and the X-ray analysis for composition, the growth rate was separated into its constituent binaries. Fig. 4 indicates an enhanced indium incorporation rate as the substrate temperature increases. This is in marked contrast to the sharply decreasing indium mole fraction in InGaP which is found as the temperature increases [8]. A similar trend toward higher In incorporation is observed in the

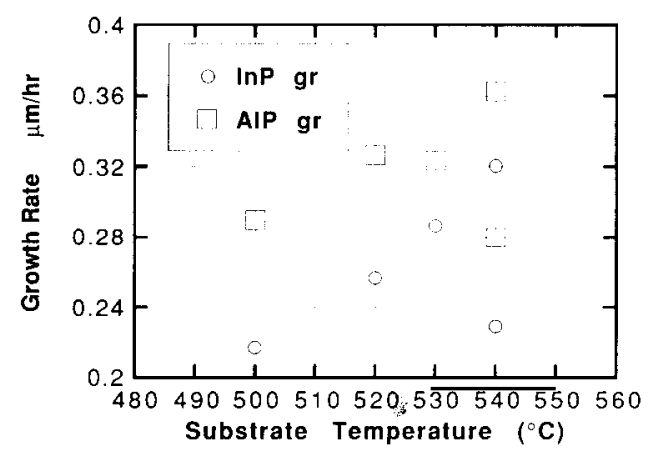

Fig. 4. Growth rate of constituent binaries InP and AIP versus substrate temperature for constant hydride cracking temperature, group V flow and group III flow. growth of InAlAs from 530 to $650^{\circ} \mathrm{C}$ [7], and for a part of the temperature range for InGaAs [9]. This trend of increasing growth rates for both the InP and AlP growth rates may indicate a reaction rate limited regime. The dichotomy in the higher temperature portion of the graph is under investigation. For the growth of InAlP at higher and lower substrate temperatures, no $X$-ray signal from the epi-material is discernible and the surface morphology becomes very rough. Initial attempts to improve the material characteristics and widen the growth window by increasing hydride cracking temperature and varying the V/III ratio have not been successful.

\section{Conclusions}

InAIP has been grown for the first time using trimethyl amine alane in a CBE system. Carbon levels are more than an order of magnitude less than those found in material grown by CBE using triethyl or trimethyl aluminum. While oxygen levels are higher than desired, they may be reduced by using drying filters in the hydride line. The quality of the films is very sensitive to substrate temperature with relatively smooth surfaces and good X-ray characteristics confined to $535-545^{\circ} \mathrm{C}$. The X-ray FWHM is comparable to that grown by GSMBE.

\section{Acknowledgements}

This work was supported by the US Army Research Office, URI Program, Contract DAAL 03-86-K-0007.

\section{References}

[1] J. Hashimoto, T. Katsuyama, J. Shinkai, I. Yoshida and H. Hayashi, Appl. Phys. Letters 58 (1991) 879.

[2] D.P. Bour and J.R. Shealy, J. Quantum Electron. QE-24 (1988) 1856.

[3] J.A. Varriano, M.W. Koch, F.G. Johnson and G.W. Wicks, J. Electron. Mater. 21 (1992) 195

[4] M.J. Hafich, H.T. Lee, G.Y. Robinson, D. Li and N. Otsuka, J. Appl. Phys. 69 (1991) 752. 
[5] T.J. de Lyon, J.M. Woodall, P.D. Kirchner, D.T. McInturff, G.J. Scilla and F. Cardone, J. Vacuum Sci. Technol. B 9 (1991) 136.

[6] C.R. Abernathy, A.S. Jordan, S.J. Pearton, W.S. Hobson, D.A. Bohling and G.T. Muhr, Appl. Phys. Letters 56 (1990) 2654.
[7] M.E. Sherwin and G.O. Munns, J. Vacuum Sci. Technol. B 10 (1992) 943.

[8] J.C. Garcia, P. Maurel, P. Bove and J.P. Hirtz, J. Appl. Phys. 69 (1991) 3297.

[9] N. Kobayashi, J.L. Benchimol, F. Alexandre and Y. Gao, Appl. Phys. Letters 51 (1987) 1907. 